\title{
UMOLHARÀ LUZ DA BIOÉTICA PRINCIPIALISTA NO SERVIÇO DE ATENDIMENTO MÓVEL DE URGÊNCIA
}

Benedito Fernandes da Silva Filho ${ }^{1}$ Caroline Borges Duque ${ }^{1}$ Rita Narriman Silva de Oliveira Boery ${ }^{1}$ Sérgio Donha Yarid ${ }^{1}$

\author{
http://orcid.org/0000-0003-2464-9958 \\ http://orcid.org/0000-0003-0961-1097 \\ http://orcid.org/0000-0002-7823-9498 \\ http://orcid.org/0000-0003-0232-4212
}

Objetivo: Refletir acerca da aplicabilidade dos princípios da Bioética Principialista no trabalho do enfermeiro que atua no Serviço de Atendimento Móvel de Urgência. Metodologia: Trata-se um estudo reflexivo teórico-conceitual baseado na Bioética Principialista e na Declaração sobre Ética dos Serviços Médicos de Urgência, relacionando-os com as condutas do enfermeiro no serviço. Resultados: Elencaram-se seis tópicos para discussão e, com isso, identificaram-se os Princípios Bioéticos que embasam o atendimento às vítimas. Além disso, buscou-se como tais padrões de condutas norteiam o atendimento aos usuários em relação às intervenções do enfermeiro, impactando diretamente na qualidade assistencial. Conclusão: O desenvolvimento do processo de trabalho do enfermeiro regulado na Bioética Principialista distingue o quanto é importante o desenvolvimento de virtudes associadas às competências e habilidades técnicas. O compromisso e a responsabilidade Bioética não devem ser negligenciadas no atendimento dos serviços de saúde em especial aos da urgência, como no Serviço de Atendimento Móvel de Urgência.

Descritores: Bioética; Ética Baseada em Princípios; Serviços Médicos de Emergência; Ambulância; Enfermagem.

\section{A LOOK IN THE LIGHT OF PRINCIPLE BIOETHICS AT THE MOBILE EMERGENCY CARE SERVICE}

Objective: To reflect on the applicability of the principles of Bioethics in the work of nurses working in the Mobile Emergency Care Service. Methodology: This is a theoretical-conceptual reflective study based on the Principle Bioethics and the Declaration of Ethics of the Emergency Medical Services, relating them to the conduct of nurses in the service. Results: We listed six topics for discussion and thus identified the Bioethical Principles that underlie the care of victims and know how they guide the care of users in relation to nurse interventions, reflecting directly on the quality of care. Conclusion: The development of the regulated nurse work process in Principle Bioethics distinguishes how important is the development of virtues associated with technical competencies and skills. Bioethical commitment and responsibility should not be neglected in the care of health services, especially those of urgency as in the Mobile Emergency Care Service.

Descriptors: Bioethics; Principle-Based Ethics; Emergency Medical Service; Ambulances; Nursing.

\section{UNA MIRADAA LA LUZ DE PRINCIPLE BIOETHICS EN EL SERVICIO MÓVIL DE ATENCIÓN DE EMERGENCIA}

Objetivo: Reflexionar sobre la aplicabilidad de los principios del Principio de Bioética en el trabajo de las enfermeras que trabajan en el Servicio Móvil de Atención de Emergencia. Metodología: Este es un estudio reflexivo teórico-conceptual basado en el Principio de Bioética y la Declaración de Ética de los Servicios Médicos de Emergencia, relacionándolos con la conducta de las enfermeras en el servicio. Resultados: Enumeramos seis temas para el debate y, por lo tanto, identificamos los Principios bioéticos que subyacen a la atención de las víctimas y saber cómo guían la atención de los usuarios en relación con las intervenciones de enfermería, reflejando directamente en la calidad de la atención. Conclusión: El desarrollo del proceso de trabajo de enfermería regulado en Principio Bioética distingue la importancia del desarrollo de las virtudes asociadas con las competencias y habilidades técnicas. El compromiso y la responsabilidad bioética no deben descuidarse en la atención de los servicios de salud, especialmente los de urgencia como en el Servicio móvil de atención de emergencia.

Descriptores: Bioética; Ética Basada en Principios; Servicios Médicos de Urgencia; Ambulancias; Enfermería.

${ }^{1}$ Universidade Estadual do Sudoeste da Bahia (UESB), Jequié, Bahia.

Autor correspondente: Benedito Fernandes da Silva Filho - E-mail: ditofilho13@gmail.com 


\section{INTRODUÇÃO}

Por volta do ano 2000, foi implantado, no Brasil, pelo Governo Federal, o Serviço de Atendimento Móvel de Urgência (SAMU), considerado o principal componente móvel da rede de atenção às urgências. Por ora, o principal objetivo do SAMU é reduzir as sequelas decorrentes da demora no atendimento e o tempo de hospitalizações, bem como, reduzir de forma significativa o número de óbitos ${ }^{(1)}$.

O SAMU dispõe-se sobre um modelo assistencial, o qual tem padronização operativa regulada por meio de uma central telefônica com discagem gratuita pelo número 192, além de possuir uma regulação médica descentralizada, hierarquizada e regionalizada. Há, assim, um sistema de atendimento de socorro normatizado, mediante a complexidade das situações de emergência que definirão os tipos de unidade e a atribuição de recursos que serão utilizados.

Desta forma, como uma modalidade de assistência emergencial que ganha destaque pelas suas particularidades, o SAMU realiza o Atendimento Pré-hospitalar (APH) precocemente à vítima, com chegada posterior à ocorrência de uma injúria à sua saúde de diversas naturezas, entre elas: cirúrgica, clínica, traumática, psiquiátrica, obstétrica, pediátrica, dentre outras, as quais possam provocar sequelas, sofrimentos, ou até mesmo óbitos, sendo considerado um atendimento indispensável. Portanto, oferta-se à vítima atendimento e/ou transporte adequado a um serviço integrado ao Sistema Único de Saúde (SUS) ${ }^{(1.2)}$.

O SUS foi promulgado pela Constituição Federal de $19888^{(3)}$ e ratifica a saúde como um direito universal. Destaca-se que, de acordo com essa universalidade de acesso, existem inúmeras situações que geram reflexões e/ou conflitos éticos e bioéticos que merecem discussão para uma abordagem assistencial mais humanizada.

Nesse sentido, a bioética estuda a moralidade da conduta humana no campo das ciências da vida. Assim, pode ser relacionada como a ética dos profissionais de saúde no seu exercício laboral, pela sua relação com o processo saúde/doença dos seres humanos. Também denominada de ética biomédica, perpassa pelos dilemas morais relacionados à prática biomédica frente ao desenvolvimento tecnológico e às questões político-sociais ${ }^{(4)}$.

Com a publicação do documento Belmont Report, percebe-se que as concepções bioéticas expandiram-se na área biomédica, principalmente no campo da pesquisa, propondo, inicialmente, três princípios fundamentais: respeito à autonomia, beneficência e justiça. Acredita-se que por uma melhor discussão ética no campo da clínica assistencial, surgiu, em 1979, a publicação do livro Principles of biomedical ethics, ampliando os três princípios acima elencados pelo Relatório Belmont, para o quarto princípio, denominado de não maleficência. Dessa forma, estabeleceu-se o chamado Principialismo Bioético ou a Bioética Principialista ${ }^{(5)}$. Diante do exposto, o estudo tem como objetivo refletir acerca da aplicabilidade dos princípios da Bioética Principialista no trabalho do enfermeiro que atua no Serviço de Atendimento Móvel de Urgência.

\section{METODOLOGIA}

Trata-se um estudo reflexivo teórico-conceitual construido a partir de inquietações profissionais sobre a aplicação da bioética no serviço de Urgência pelo enfermeiro. No intuito de fundamentar a reflexão, utilizaram-se os pilares da Bioética Principialista e da Declaração sobre Ética dos Serviços Médicos de Urgência, relacionando-os com as condutas do enfermeiro no APH por meio do SAMU.

Desse modo, a seguinte reflexão está organizada de acordo com seis tópicos: Rotina do enfermeiro no SAMU, baseado na vivência profissional na Regional Camaçari, Bahia; Perspectivas da Bioética Principialista; Princípio da Autonomia; Princípio da Beneficência; Princípio da Não-Maleficência e Princípio da Justiça.

\section{Rotina do enfermeiro no SAMU}

O enfermeiro, após assumir o plantão, realiza as atividades de rotina e aguarda o disparo para os atendimentos das solicitações oriundas da central de regulação do SAMU. Na Regional Camaçari, o enfermeiro é parte integrante da Equipe da Unidade de Suporte Avançado de Vida, juntamente com o condutor socorrista e o médico intervencionista. A saída da Unidade de Suporte Avançado - USA ocorre mediante autorização da central de regulação.

No local do atendimento, é realizada a abordagem para estabilização ou remoção, tendo, a todo momento, o médico regulador orientando as demandas técnicas e gestoras proveniente do atendimento. Nos atendimentos realizados pela USA, visando ao menor tempo-resposta, se o atendimento evoluiu para estabilização do paciente e indicação de remoção, o médico ou o enfermeiro informa ao regulador o atual quadro clínico do paciente e, com base nestes dados, o médico regulador tomará sua decisão e fará contato com o serviço especializado adequado, oficializando a regulação da vaga.

Com a vaga autorizada, a USA dirige-se ao destino de referência, entregando o paciente aos profissionais responsáveis, seguindo todas as recomendações do protocolo e normas da regulação. No retorno à base, deve concluir as anotações necessárias na ficha de atendimento, a qual equivale ao prontuário do paciente de qualquer estabele- 
cimento de saúde, higienização da ambulância e reposição dos materiais e medicamentos utilizados ${ }^{(6)}$

Embora as atividades do cuidado sejam predominantes, o enfermeiro do SAMU Regional Camaçari, desenvolve ações gerenciais e educativas. Corroborando com a pesquisa de Luchtemberg e Pires: “as ações de cuidado são diversificadas, predominando ações de cuidado direto aos pacientes/usuários, as quais vão desde a avaliação da cena da ocorrência, até a realização de diversos procedimentos (7:217)". Ainda, na dimensão do cuidar, os acompanhes/familiares são acolhidos e, quando necessário, atendidos pela equipe.

No processo de trabalho do enfermeiro, as atividades administrativo/gerencial não se dissociam das assistenciais, sendo realizadas todas as ações de provisão de materiais e suprimentos para ambulâncias, preenchimento de formulários e outros impressos essenciais para o controle e avaliação do serviço. O papel educativo é realizado na dimensão das orientações a toda comunidade sobre o funcionamento e acionamento coerente do serviço.

\section{Perspectivas da Bioética Principialista}

Tendo como fundamentação a bioética de Potter que reporta às intervenções do homem sobre a vida e a natureza e a de Helleghers que elucidava as influências da ciência sobre a saúde do indivíduo, o primeiro modelo de bioética surge, concomitante, com a necessidade de orientação na conduta frente aos dilemas éticos, especialmente nas ciências médicas, originado os quatro princípios (autonomia, beneficência, não-maleficência e justiça). Assim, denomina-se Bioética Principialista ${ }^{(4)}$.

Baseada na Bioética Principialista, a Declaração sobre a Ética dos Serviços Médicos de Urgência ${ }^{(9)}$, aprovada por unanimidade pelos participantes durante II Jornada de Emergência Médica, em Lisboa, em 7 de dezembro de 1990, estabeleceu que os Sistemas Mundiais de Ajuda Médica de Urgência devem obedecer aos principios fundamentais dos Direitos do Homem. Deste modo, busca-se reportar esses quatros Princípios no APH realizado pelo SAMU

\section{Principio da Autonomia}

O trabalho do enfermeiro no SAMU é caracterizado por constantes desafios que geram oportunidades de aprendizado e satisfação, mas, por se tratar de um serviço de urgência, é cercado de ações de alta complexidade, gerando situações de estresse e possibilidade de negligência ao princípio da autonomia.

Trata-se do primeiro princípio citado pela Declaração Ética dos Serviços Médicos de Urgência de Lisboa ${ }^{(9)}$, de- vendo a autonomia do usuário ser respeitada de maneira absoluta, sua liberdade de escolha mesmo nos casos de urgências. Além disso, destaca-se que os cuidados prestados devem valorizar a autonomia dos usuários no sentido físico-psico-social. No entanto, observa-se que tal valorização nem sempre é praticável pelos profissionais de saúde, principalmente, quando o usuário se encontra numa unidade de internação intensiva ${ }^{(9)}$.

Não obstante, é notório que enfermeiro do SAMU ao realizar o atendimento primário ou secundário, deve respeitar a autonomia do paciente/usuário em todos os procedimentos, no transporte, e até mesmo na recusa do atendimento. Entretanto, deve identificar as alterações de comportamento que indiquem se o paciente está prejudicado em sua capacidade de decisão, como a intoxicação etílica ou por outras drogas, as quais causem alterações do nível de consciência. Cabe ao enfermeiro e equipe explicar a importância do atendimento para o restabelecimento de sua condição de saúde, tranquilizando-o. Na perseverança da recusa, deve ser oficializada na ficha de atendimento com assinatura do paciente/ usuário ou alguma testemunha( ${ }^{(6)}$.

\section{Principio da Beneficência}

A vivência como enfermeiro no SAMU Regional Camaçari permitiu compreender o quanto é importante e especializado o Atendimento Pré-Hospitalar. Para atuar no SAMU, o enfermeiro necessita de conhecimento e habilidade técnica específica para prestar um atendimento dinâmico e eficiente. Esta responsabilidade está voltada à beneficência. Isso significa que todo ato ético deve beneficiar tanto o enfermeiro como o paciente. Neste contexto, a beneficência reporta-se a uma ação realizada em benefício do outro, em promover ou fazer o bem ${ }^{(4)}$.

No SAMU, o enfermeiro executa o atendimento baseado no princípio da beneficência, visando sempre o benefício do paciente/usuário, tendo como base a Declaração Ética dos Serviços Médicos de Urgência de Lisboa ${ }^{(9)}$, com destaque ao dever de maximizar os benefícios de saúde e alcançar a melhor qualidade assistencial. A beneficência também é considerada na disponibilidade de pontos de atenção para receber, de forma efetiva, o paciente vítima de urgências, configurando-se, desta forma, o dever do Estado em garantir a segurança e promover a saúde do cidadão. Destacam-se, como pontos de apoio, as Unidades Hospitalares, Centros de Orientação de Doentes Urgentes, Serviços Móveis de Emergência e Reanimação, dentre outros que assegurem a assistência necessária e efetiva durante 24 horas ${ }^{(9)}$ 
Aqueles que solicitaram ou receberam o atendimento pautado na beneficência conhecem a dimensão de seu significado. Somente o paciente/usuário que sofreu um agravo pode dimensionar o quanto ser objeto da beneficência foi decisivo para sua sobrevivência e a minimização de sequelas. Tal perspectiva é possivel, atendendo às seguintes regras básicas: proteger e defender o direito do outro; evitar que outros sofram danos; eliminar as condições que causarão danos; ajudar pessoas inaptas e socorrer as pessoas que estão em perigo ${ }^{(10)}$.

\section{Principio da Não-Maleficência}

No atendimento ao paciente/usuário, o enfermeiro do SAMU deve usar o acolhimento como estratégia de estabelecimento de vínculo. É muito importante apresentar-se, informar que está ali para ajudar, emitindo palavras de encorajamento e otimismo. A não-maleficência na comunicação enfermeiro-paciente é a ponte entre os procedimentos e as explicações, minimizando prejuízo aos agravos/à doença em si. Além disso, o entendimento que o princípio da não-maleficência se refere ao comprometimento de evitar causar danos e prejuízos ${ }^{(4)}$ é um reconhecimento da sua homogeneidade com o princípio da beneficência.

O terceiro princípio da Declaração Ética dos Serviços Médicos de Urgência de Lisboa ${ }^{(9)}$ destaca que os profissionais de saúde devem reduzir, ao mínimo possível, os prejuízos resultantes de procedimentos necessários nas situações de urgência, pois tratam-se de procedimentos com maior grau de risco.

Nessa perspectiva, guiado pelo princípio da não-maleficência, o enfermeiro do SAMU não pode realizar ações e/ ou transportes sem as condições de trabalho necessários para o desenvolvimento de suas atividades profissionais. O trabalho do enfermeiro do SAMU deve estar pautado na competência e em habilidades técnicas, numa comunicação efetiva e, por fim, na preocupação com a acomodação e transporte, desta maneira, atendendo as expectativas do paciente e acompanhantes/familiares. Vale ressaltar que a atuação do enfermeiro do SAMU está pautada em deveres definidos no Código de Ética dos Profissionais de Enfermagem, como a prestação de uma assistência de Enfermagem isenta de danos procedentes de imperícia, negligência ou imprudência ${ }^{(8)}$, os quais estão fundamentadas no princípio hipocrático "primeiro não causar dano" do qual deriva o princípio de não-maleficência ${ }^{(9)}$.

\section{Principio da Justiça}

O enfermeiro, como profissional integrante do APH do SAMU, pode vivenciar o principio da justiça ao realizar o acolhimento, a classificação de risco e a intervenção adequada, correlacionando com o quarto princípio da Declaração Ética dos Serviços Médicos de Urgência de Lisboa ${ }^{(9)}$, que visa a distribuição de ajuda de forma justa, ofertando assistência a todos que tenham necessidade.

O princípio da justiça ocupa um papel protagonista no SAMU, uma vez que o envio do recurso e o atendimento em lócus deve obedecer a equidade na distribuição de recursos, na intervenção e na igualdade de acesso aos serviços de referência. A igualdade de direitos entre os indivíduos estabelece que os enfermeiros do SAMU realizem o máximo de atendimentos de urgência ao maior número de pacientes/usuários que deles necessitam, com qualidade. $\mathrm{Na}$ parte gerencial, o enfermeiro desenvolve atividades que visam uma otimização dos recursos materiais e equipamentos, oportunizados a continuidade do APH.

O princípio de justiça envolve uma série de questões de ordem, social, política e econômica. Portanto, vislumbra um princípio polêmico e complexo para vários autores. Por ora, o que fica evidente é a concepção, para o enfermeiro, que o SAMU e o atendimento realizado por ele, deve pautar-se no respeito, honestidade e na justiça.

\section{Limitações do estudo}

Destaca-se como limitação a impossibilidade de promover uma discussão direta com resultados de estudos, de outros autores, em virtude da escassez de estudos que abordam a temática em um serviço móvel de urgência.

\section{Contribuições do estudo para a prática}

Pode-se considerar que esse é um estudo pioneiro e atual que traz a aplicabilidade dos princípios da Bioética Principialista no trabalho do enfermeiro que atua no Serviço de Atendimento Móvel de Urgência. Nesta perspectiva, a presente reflexão vislumbra melhorar a compreensão acerca da utilização dos princípios da bioética principialista no trabalho do enfermeiro frente ao SAMU. Espera-se que as reflexões contidas nesta pesquisa agreguem valores ao trabalho do enfermeiro e contribuam para uma melhor assistência aos pacientes atendidos pelo SAMU. Além disso, abre-se mais um espaço de debate para o campo da bioética e instiga-se que mais estudos sejam desenvolvidos na perspectiva de conhecer com maior profundidade os aspectos bioéticos envolvidos e como os enfermeiros realizam a aplicabilidade de seus princípios.

\section{Considerações finais}

$\mathrm{Na}$ presente reflexão, observou-se que o processo de trabalho do enfermeiro que atua no APH do SAMU, pautado 
na bioética principialista, evidencia o quanto é importante o desenvolvimento de virtudes, associando-as às competências e habilidades técnicas. O compromisso e a responsabilidade bioética não devem ser negligenciadas no atendimento dos serviços de saúde, em especial, nos da urgência como no APH do o SAMU.

Torna-se evidente que o enfermeiro respeita o paciente/usuário como cidadão e aos seus direitos, garantindo o acolhimento e a classificação de risco no atendimento. Destaca-se, também, o cuidado no amparo aos familiares dos usuários que estão envolvidos emocionalmente no evento, por este ser inesperado e imbuído de tensão.

A qualidade do atendimento prestado e as ações do enfermeiro devem ter como finalidade principal trazer benefícios para o usuário, ao promover um cuidado dinâmico, eficiente, seguro e sem danos. Ao realizar atualização

\section{REFERÊNCIAS}

1. Almeida PMV, Dell'Acqua MCQ, Cyrino CMS, Juliani CMCM, Palhares VC, Pavelqueires S. Análise dos atendimentos do SAMU 192: Componente móvel da rede de atenção às urgências e emergências. Esc Anna Nery. [Internet] 2016 [cited 2019 May 5]; 20 (2): 289-295. Available from: http://www.scielo. br/pdf/ean/v20n2/1414-8145-ean-20-02-0289.pdf

2. Teles AS, Coelho TCB, Ferreira MPS, Scatena JHG. Serviço de Atendimento Móvel de Urgência (SAMU) do Estado da Bahia: subfinanciamento e desigualdade regional. Cad Saúde Colet. [Internet] 2017 [cited 2019 Oct 27]; 25 (1): 51-57. Available from: http://www.scielo.br/pdf/cadsc/v25nl/1414-462Xcadsc-25-1-51.pdf

3. Macedo VLM, Vieira LF, Neves RS, Suderlan SL. Avaliação da estratégia saúde da familia em São Sebastião - Distrito Federal. Enferm Foco. [Internet] 2019 [cited 2019 Oct 27]; 10 (2): 15-21. Available from: https://docs.google.com/ viewerng/viewer?url=http://revista.cofen.gov.br/index.php/ enfermagem/article/viewFile/2330/540

4. Souza Junior EV, Silva VSB, Lozado YA, Bomfim ES, Alves JP, Boery EN, Boery EN. Dilemas bioéticos na assistência médica às gestantes adolescentes. Rev Bioét. [Internet] 2018 [cited 2019 Oct 27]; 26 (1): 87-94. Available from: http:// www.scielo.br/scielo.php?script=sci_arttextEpid=S1983$80422018000100087 \varepsilon$ lng=en\&nrm=iso\&tlng=pt

5. Garrafa V, Martorell LB, Nascimento WF. Críticas ao principialismo em bioética: perspectivas desde o Norte e desde o Sul. Saúde soc. [Internet] 2016 [cited 2019 Oct 27]; 25 (2): 442-451. Available from: http://www.scielo.br/scielo. php?script=sci_arttextEpid=S0104-12902016000200442

6. Ministério da Saúde(BR). Secretaria de Atenção à Saúde. Departamento de Atenção Especializada. Regulação médica profissional técnica e em bioética para assistência de enfermagem no APH do SAMU, o enfermeiro passa a se conscientizar que sua atuação assistencial, gerencial e educativa são os alicerces de sua prática profissional.

Evidencia-se a necessidade de estudos com o intuito de elucidar o trabalho do enfermeiro no APH do SAMU, em especial, o entendimento dos princípios bioéticos aplicados a sua atuação. O maior detalhamento de estudos nesta temática poderá contribuir para promoção de ações efetivas, melhorando a qualidade da assistência prestada pelos profissionais que atuam no SAMU

Contribuições dos autores: BFSF, CBD: concepção e desenho; análise, interpretação dos dados e redação do artigo: RNSOB, SDY: revisão crítica e revisão final.

Conflito de Interesse: não há.

das urgências / Ministério da Saúde, Secretaria de Atenção à Saúde, Departamento de Atenção Especializada. - Brasilia: Editora do Ministério da Saúde. [Internet] 2006 [cited 2019 Aug 27]. Available from: http://bvsms.saude.gov.br/bvs/ publicacoes/regulacao_medica_urgencias.pdf

7. Luchtemberg MN, Pires DEP. Enfermeiros do Serviço de Atendimento Móvel de Urgência: perfil e atividades desenvolvidas. Rev Bras Enferm. [Internet] 2016 [cited 2019 Aug 27]; 69 (2): 213-220. Available from: http://www.scielo.br/scielo. php?script=sci_arttext\&pid=S0034-71672016000200213\&lng $=p t \& t$ lng=pt

8. Conselho Federal de Enfermagem (COFEN). Resolução COFEN № 0564/2017. Aprova o novo Código de Ética dos Profissionais de Enfermagem [Internet]. 2017 [cited 2019 Aug 29]; Available from: http://www.cofen.gov.br/resolucao-cofenno-5642017_59145. html

9. Declaração de Lisboa. Conclusões e recomendações das II Jornadas de Emergência Médica de Lisboa. [Internet] 1990 [cited 2019 Aug 27]. Available from: http://bvsms.saude.gov. br/bvs/publicacoes/declaracao_lisboa_etica_urgencia_ medica.pdf

10. Cogo SB, Lunardi VL, Quintana AM, Girardon-Perlini NMO, Silveira RS. Assistência ao doente terminal: vantagens na aplicabilidade das diretivas antecipadas de vontade no contexto hospitalar. Rev Gaúcha Enferm. [Internet] 2017 [cited 2019 Oct 27]; 38 (4): e65617. Available from: http:// www.scielo.br/scielo.php?script=sci_arttext\&pid=S1983$14472017000400418 \varepsilon$ lng $=$ en

RECEBIDO 28/9/2019

ACEITO - 04/01/2020 